\title{
Clinical implications of tenascin-C and OX40 ligand in patients with acute coronary syndrome
}

\author{
JIN-HU YANG ${ }^{1}$ and FENG REN ${ }^{2}$ \\ Departments of ${ }^{1}$ General Surgery and ${ }^{2}$ Clinical Laboratory, The Fourth People's Hospital of Wuxi, \\ Wuxi, Jiangsu 214062, P.R. China
}

Received September 24, 2013; Accepted October 25, 2013

DOI: $10.3892 /$ br.2013.195

\begin{abstract}
Acute coronary syndrome (ACS) typically occurs when coronary artery disease results in the obstruction of the coronary arteries. Tenascin-C (TNC) and OX40 ligand (OX40L) were shown to be involved in the pathogenesis of atherosclerosis. In this study, 50 healthy controls and 170 patients, including 50 patients with stable angina (SA), 70 with unstable angina and 50 with acute myocardial infarction, were evaluated to assess serum TNC and plasma OX40L levels. The serum TNC levels were measured by a quantitative automated particle-enhanced immunonephelometric assay. ELISA was used to determine the expression levels of OX40L. All the coronary stenoses with a $\geq 30 \%$ diameter reduction were assessed by angiographic coronary stenosis morphology. The patients with ACS exhibited a significant increase in TNC expression levels $(39.39 \pm 19.80 \mathrm{ng} / \mathrm{ml})$ compared to the levels in the control and SA groups $(28.65 \pm 12.32 \mathrm{ng} / \mathrm{ml}, \mathrm{P}<0.01$ and $31.22 \pm 18.92 \mathrm{ng} / \mathrm{ml}, \mathrm{P}<0.05$, respectively). The levels of OX40L were also found to be higher in patients with ACS $(38.59 \pm 15.76 \mathrm{ng} / \mathrm{ml})$ compared to those in the control and SA groups $(19.42 \pm 11.19 \mathrm{ng} / \mathrm{ml}, \mathrm{P}<0.001$ and $21.52 \pm 10.30 \mathrm{ng} / \mathrm{ml}$, $\mathrm{P}<0.001$, respectively). The TNC and OX40L levels were positively correlated with each other $\left(\mathrm{r}_{1}=0.68 ; \mathrm{P}<0.001\right)$ and with fibrinogen levels $\left(r_{3}=0.76\right.$ and $r_{4}=0.45$, respectively; $\mathrm{P}<0.001)$. A positive correlation was also observed between the expression of TNC and OX40L and complex coronary stenosis $\left(r_{5}=0.69\right.$ and $r_{6}=0.55$, respectively; $\left.P<0.001\right)$. We concluded that TNC and OX40L may act synergistically in coronary plaque formation and may be also be involved in the pathogenesis of coronary lesions. Patients with ACS exhibited increased TNC and OX40L expression levels, which may have created a prothrombotic milieu, aggravating the development of atherosclerosis and the instability of atherosclerotic plaques. Therefore, the expression of TNC and OX40L may be a valuable marker for predicting the severity of ACS.
\end{abstract}

Correspondence to: Dr Feng Ren, Department of Clinical Laboratory, The Fourth People's Hospital of Wuxi, 200 Huihe Road, Wuxi, Jiangsu 214062, P.R. China

E-mail: renfeng198254@163.com; goldtiger007@126.com

Key words: tenascin-C, OX40 ligand, acute coronary syndrome

\section{Introduction}

Acute coronary syndrome (ACS) typically occurs when coronary artery disease (CAD) results in the obstruction of coronary arteries. This may lead to myocardial infarction (MI), heart failure (HF), arrhythmias, cardiac arrest and death $(1,2)$. The current clinical practice includes rapid patient evaluation and risk stratification based on clinical and electrocardiographic characteristics, as well as the assessment of biochemical markers (3). Cardiovascular diseases, such as MI, hypertension, hypertrophy or $\mathrm{HF}$, are accompanied by changes in the composition of the cardiac extracellular matrix (ECM). These dynamic alterations may determine the mechanical properties of the damaged heart and directly modulate the inflammatory and reparative response (4).

Tenascin-C (TNC) is a multifunctional hexameric glycoprotein that is a major component of the ECM. It is synthesized by interstitial fibroblasts and its levels are increased in inflammatory diseases. Upon tissue damage, TNC has a multitude of functions that mediate inflammatory and fibrotic processes, in order to enable effective tissue repair. Over the last decade, accumulating evidence indicated a vital role for TNC in cardiac and arterial injury, tumor angiogenesis and metastasis, as well as in the modulation of stem cell behavior (5). Previous in vitro studies demonstrated that a range of factors implicated in cardiovascular disease appear to be able to stimulate TNC synthesis by fibroblasts $(6,7)$.

OX40, also referred to as CD134 or TNFRSF4, is a member of the tumor necrosis factor (TNF) co-stimulatory receptor family and is expressed on activated T cells. OX40 is specifically upregulated by the human T-lymphotropic virus type I (HTLV-1) viral transactivator, Tax. The ligand of OX40 (OX40L), which belongs to the TNF superfamily, was first identified as glycoprotein 34 on HTLV-1-transformed cells and was found to bind OX40. OX40-OX40L interactions may affect the activity and differentiation of several types of immune cells (8). It was recently demonstrated that genetic variants in the OX40L locus are associated with $\mathrm{MI}$ and the severity of CAD in humans. OX40-OX40L pairs were shown to be involved in ACS (9).

Willett et al (10) demonstrated that the enforced trimerization of feline CD134L (OX40L) via the introduction of a subdomain of the TNC oligomerization domain, was able to restore full ligand-binding activity. The resulting fusion 
protein was proven to be as effective as the anti-CD134 monoclonal antibody in detecting CD134 expression and displayed a strain-specific blocking of viral entry. Therefore, we surmised that the interaction of TNC and OX40L is important in atherosclerosis. In the present study, we investigated the expression of serum TNC and OX40L and assessed the correlation between their expression and the number of complex lesions in the patients. In addition, the statistical correlation between TNC and OX40L or fibrinogen was evaluated.

\section{Patients and methods}

Subjects. The patients and healthy controls were involved in the present study over a period of 18 months. Patients undergoing clinically indicated diagnostic coronary angiography in the coronary care unit of The Fourth People's Hospital of Wuxi, were consecutively registered. The patients were classified into the ACS and the stable angina (SA) groups. The ACS group consisted of patients with unstable angina (UA) and acute myocardial infarction (AMI). The patients with UA experienced ischemic chest pain at rest within the preceding $48 \mathrm{~h}$, without enzymatic evidence of myocardial necrosis; the AMI patients were defined as those with an occurrence of MI within the preceding 4 weeks. The diagnosis of MI was based on typical chest pain and ST segment elevation in at least two contiguous electrocardiographic leads. The patients with SA $(n=50)$ underwent coronary angiography due to signs and symptoms of clinical SA. For comparison, 50 gender- and age-matched donors served as the control group. Patients with concurrent infection, tumor, liver or kidney diseases were excluded (Table I). All the patients provided written informed consent prior to their inclusion in the study. This study was approved by the ethics committee of the Affiliated Hospital of Jiangnan University.

Blood sampling protocol. Peripheral venous blood samples were drawn into blood collection tubes using a suction catheter (BD Vacutainer, Franklin Lakes, NJ, USA). The blood to be used for the assessment of serum TNC levels was collected in tubes containing coagulant and sent to the clinical laboratory. The blood to be used for the assessment of plasma OX40L levels was collected in tubes containing $\mathrm{Na}_{2}$-EDTA at a final concentration of $0.1 \%$ and immediately centrifuged at $1,000 \mathrm{x} \mathrm{g}$ for $10 \mathrm{~min}$ at $4^{\circ} \mathrm{C}$. The supernatant was stored at $-80^{\circ} \mathrm{C}$ until analysis. The samples were thawed only once.

The serum TNC levels were measured by a quantitative automated particle-enhanced immunonephelometric assay (Boehringer Mannheim, Mannheim, Germany). The plasma levels of OX40L were determined using a rat anti-human-OX40L ELISA kit (Assay Designs, Inc., Ann Arbor, MI, USA) according to the manufacturer's instructions. Additional laboratory measurements were performed using standard methods. Fibrinogen was measured using the clotting method (11).

Coronary angiography. Coronary angiography was performed using a femoral approach with 6-Fr diagnostic catheters (Cordis Corp., FL, USA). Images were recorded in multiple projections for the right and left coronary arteries. All the coronary stenoses with a $\geq 30 \%$ diameter reduction were assessed by two experienced cardiologists who were blinded to the levels of TNC and OX40L, as well as to the identity and clinical characteristics of the patients. Complex lesions were defined by the following characteristics: i) irregular morphology, or scalloped borders, or both; ii) overhanging or abrupt edges perpendicular to the vessel wall; iii) ulceration; and/or iv) the presence of filling defects consistent with intracoronary thrombus. The Gensini's scoring system (12) was used to quantitatively assess the degree of stenosis for each coronary vascular lesion.

Statistical analysis. All the numerical data are expressed as means \pm standard deviation. Statistical evaluation was performed with GraphPad software (Prism 5.0) and SPSS software, version 11.7 (SPSS, Inc., Chicago, IL, USA). The correlation was evaluated using a regression analysis. The Spearman's two-way test was used to assess the correlation between two quantitative variables with non-normal distribution. The Pearson's two-way test was used to assess the correlation between two quantitative variables with normal distributions. $\mathrm{P}<0.05$ was considered to indicate a statistically significant difference.

\section{Results}

Subject characteristics. The demographic and clinical characteristics of the study participants are presented in Table I. The study included 170 patients with angiographically documented CAD. A total of 120 patients constituted the ACS group (70 patients with UA, without evidence of myocardial necrosis, and 50 with recent $\mathrm{MI}$ ) and 50 patients constituted the SA group. The healthy control group comprised 50 subjects. The patients with CAD were significantly older compared to the controls $(\mathrm{P}<0.01)$. The levels of total cholesterol, high-density lipoprotein (HDL), cholesterol and lipoprotein (a) were significantly different between the CAD and the control groups $(\mathrm{P}<0.001)$. The use of medications (calcium antagonists, nitroglycerine, angiotensin-converting enzyme inhibitors, $\beta$-blockers, 3-hydroxy-3-methylglutaryl-coenzyme A reductase inhibitors or aspirin) was significantly higher in the ACS and SA groups compared to that in the control group $(\mathrm{P}<0.001$ for all the medications).

Serum TNC and plasma OX40L levels. As shown in Table II, the TNC levels in the serum samples obtained from patients with ACS were significantly higher $(39.39 \pm 19.80 \mathrm{ng} / \mathrm{ml})$ compared to those obtained from patients in the control and SA groups $(28.65 \pm 12.32 \mathrm{ng} / \mathrm{ml}, \mathrm{P}<0.01$ and $31.22 \pm 18.92 \mathrm{ng} / \mathrm{ml}$, $\mathrm{P}<0.05$, respectively). The OX40L levels were also significantly higher in the plasma samples obtained from patients with ACS $(38.59 \pm 15.76 \mathrm{ng} / \mathrm{ml})$ compared to those obtained from patients in the the control and SA groups $(19.42 \pm 11.19 \mathrm{ng} / \mathrm{ml}$ and $21.52 \pm 10.30 \mathrm{ng} / \mathrm{ml}$, respectively; $\mathrm{P}<0.001$ for both differences).

Correlation between TNC and OX4OL expression. The seum levels of TNC in patients with ACS exhibited a positive correlation with the plasma levels of OX40L $\left(\mathrm{r}_{1}=0.68 ; \mathrm{P}<0.001\right.$; Fig. 1A). However, in the SA group, such a correlation was not observed $\left(\mathrm{r}_{2}=0.19 ; \mathrm{P}<0.001 ;\right.$ Fig. 1B).

Correlation of TNC and OX4OL with fibrinogen and coronary artery stenosis. In patients with ACS, the levels of TNC 
Table I. Statistical comparison of demographic and clinical characteristics of patients with CAD vs. those of healthy controls.

\begin{tabular}{|c|c|c|c|c|c|}
\hline \multirow[b]{2}{*}{ Characteristics } & \multicolumn{4}{|c|}{ Groups } & \multirow[b]{2}{*}{ P-value } \\
\hline & $\begin{array}{l}\text { Control } \\
(n=50)\end{array}$ & $\begin{array}{c}\text { SA } \\
(n=50)\end{array}$ & $\begin{array}{c}\text { UA } \\
(n=70)\end{array}$ & $\begin{array}{c}\text { AMI } \\
(n=50)\end{array}$ & \\
\hline Age (years) & $53 \pm 11.2$ & $61 \pm 9.3$ & $58 \pm 8.4$ & $65 \pm 10.2$ & $<0.01$ \\
\hline \multicolumn{6}{|l|}{ Gender } \\
\hline Male & 26 & 29 & 42 & 31 & \\
\hline Female & 24 & 21 & 28 & 19 & NS \\
\hline Total cholesterol (mmol/l) & $4.86 \pm 0.71$ & $4.91 \pm 0.86$ & $5.11 \pm 0.63$ & $5.22 \pm 1.12$ & $<0.01$ \\
\hline HDL (cholesterol mmol/l) & $2.05 \pm 0.72$ & $1.34 \pm 0.52$ & $1.14 \pm 0.65$ & $0.92 \pm 0.46$ & $<0.01$ \\
\hline Triglycerides (mmol/1) & $1.62 \pm 0.36$ & $1.52 \pm 0.42$ & $1.76 \pm 0.87$ & $1.82 \pm 0.92$ & NS \\
\hline Apolipoprotein B (g/l) & $0.80 \pm 0.31$ & $0.92 \pm 0.11$ & $0.69 \pm 0.35$ & $0.79 \pm 0.26$ & NS \\
\hline Lipoprotein (a) (g/l) & $0.58 \pm 0.10$ & $0.33 \pm 0.18$ & $0.41 \pm 0.22$ & $0.51 \pm 0.27$ & $<0.01$ \\
\hline Direct bilirubin $(\mu \mathrm{mol} / \mathrm{l})$ & $2.85 \pm 1.01$ & $2.60 \pm 0.57$ & $2.85 \pm 0.86$ & $2.59 \pm 0.75$ & NS \\
\hline Indirect bilirubin $(\mu \mathrm{mol} / \mathrm{l})$ & $13.24 \pm 2.73$ & $11.75 \pm 3.09$ & $12.59 \pm 2.47$ & $13.79 \pm 1.23$ & NS \\
\hline \multicolumn{6}{|l|}{ Medication (\%) } \\
\hline Calcium antagonist & 0 & 9 & 11 & 13 & $<0.001$ \\
\hline Nitroglycerin & 3 & 12 & 20 & 21 & $<0.001$ \\
\hline ACEI & 8 & 10 & 17 & 15 & $<0.001$ \\
\hline$\beta$-blockers & 15 & 72 & 78 & 85 & $<0.001$ \\
\hline HMG-CoA reductase inhibitors & 2 & 61 & 64 & 77 & $<0.001$ \\
\hline Aspirin & 1 & 85 & 91 & 98 & $<0.001$ \\
\hline
\end{tabular}

Data are expressed as means \pm standard deviation unless otherwise specified. CAD, coronary artery disease; SA, stable angina; UA, unstable angina; AMI, acute myocardial infarction; NS, no statistically significant differences between groups (P>0.05); HDL, high-density lipoprotein; ACEI, angiotensin-converting-enzyme inhibitor; HMG-CoA, 3-hydroxy-3-methylglutaryl-coenzyme.

Table II. Statistical comparison between patients with ACS or SA and healthy controls regarding serum TNC, OX40L and fibrinogen concentrations.

\begin{tabular}{lllll}
\hline & \multicolumn{4}{c}{ Groups } \\
\cline { 2 - 5 } Variables & Control $(\mathrm{n}=50)$ & $\mathrm{SA}(\mathrm{n}=50)$ & ACS (UA and AMI) (n=120) & P-value \\
\hline $\mathrm{TNC}(\mathrm{ng} / \mathrm{ml})$ & $28.65 \pm 12.32$ & $31.22 \pm 18.92$ & $39.39 \pm 19.80$ & $<0.05$ \\
OX40L $(\mathrm{ng} / \mathrm{ml})$ & $19.42 \pm 11.19$ & $21.52 \pm 10.30$ & $38.59 \pm 15.76$ & $<0.001$ \\
Fibrinogen $(\mathrm{g} / \mathrm{l})$ & $16.32 \pm 7.87$ & $19.00 \pm 11.17$ & $28.24 \pm 16.14$ & $<0.001$ \\
\hline
\end{tabular}

Data are expressed as means \pm standard deviation unless otherwise specified. ACS, acute coronary syndrome; SA, stable angina; TNC, tenascin-C; OX40L, OX40 ligand; UA, unstable angina; AMI, acute myocardial infarction.

and OX40L were significantly correlated with the levels of fibrinogen $\left(r_{3}=0.76\right.$ and $r_{4}=0.45$, respectively, $\left.\mathrm{P}<0.001\right)$ (Fig. 2A and B). There was a significant correlation between the number of complex lesions and the TNC $\left(\mathrm{r}_{5}=0.69 ; \mathrm{P}<0.001\right.$; Fig. 2C) and OX40L levels ( $\mathrm{r}_{6}=0.55 ; \mathrm{P}<0.001$; Fig. 2D).

\section{Discussion}

To the best of our knowledge, this study was the first to assess the correlation between serum TNC and plasma OX40L levels and angiographically demonstrated complex stenosis in patients with ACS. The results of this study demonstrated that the TNC and OX40L levels were increased as clinical severity increased from SA to ACS. We also observed that there was a positive correlation between TNC and OX40L expression and the number of complex lesions in patients with ACS.

Atherosclerosis is a multifactorial disease, in which inflammatory processes play an important role. Inflammation underlies lesion evolution at all stages, from plaque establishment to rupture and thrombosis (13-15). ACSs (UA, AMI and ischemic sudden death) may result from the disruption of atherosclerotic plaques, leading to coronary thrombosis. The 
A

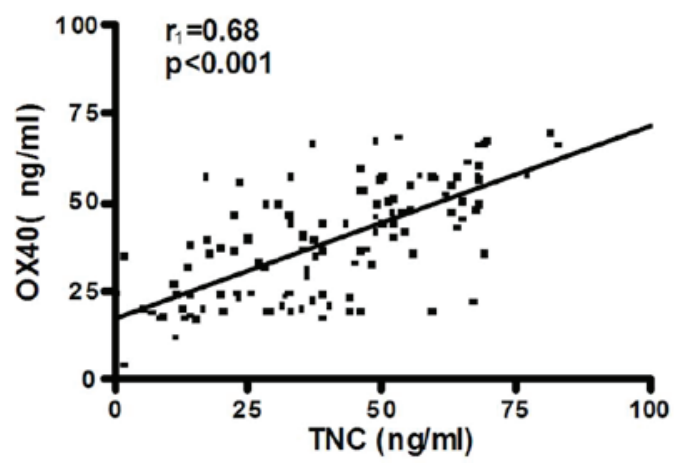

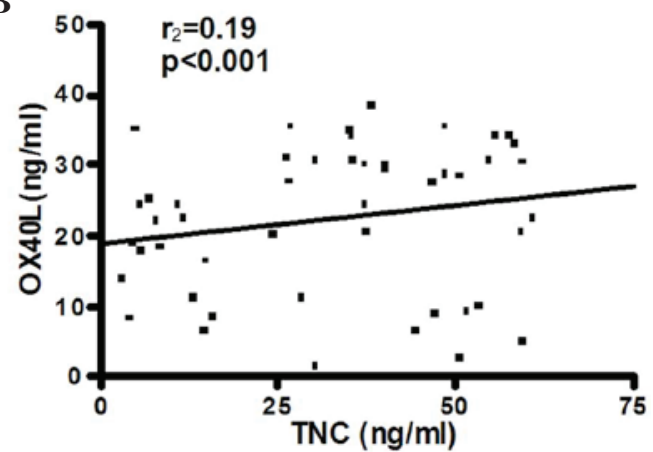

Figure 1. Correlation between the levels of serum TNC and plasma OX40L in patients with (A) acute coronary syndrome (n=120) and (B) stable angina (n=50). TNC, tenascin-C; OX40L, OX40 ligand.

A

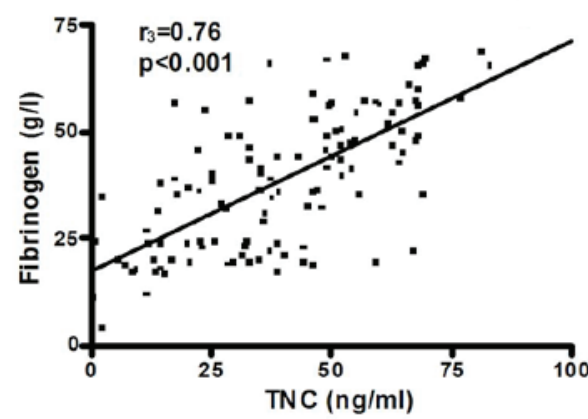

C

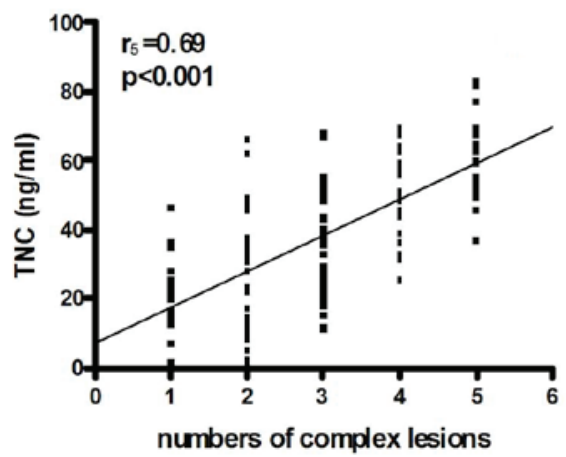

B

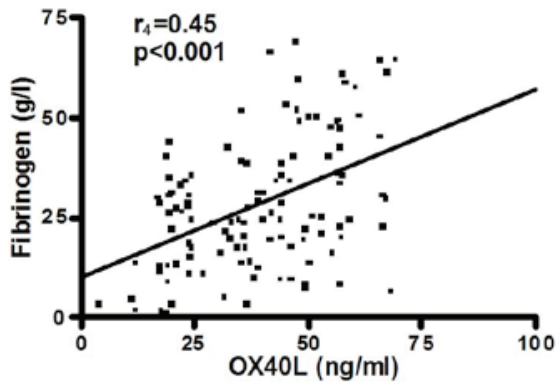

D

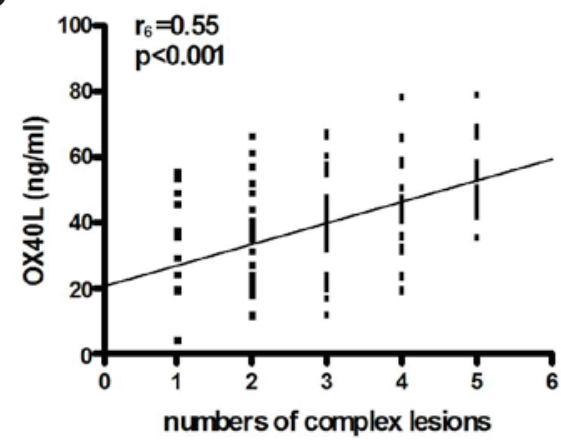

Figure 2. Correlation between the levels of (A) TNC and fibrinogen; (B) OX40L and fibrinogen; (C) TNC and coronary artery stenosis; and (D) OX40L and coronary artery stenosis. TNC, tenascin-C; OX40L, OX40 ligand.

potential cellular mechanisms involved in plaque disruption are also complex (16). We hypothesized that the levels of TNC and OX40L in patients with ACS reflect the activity of endothelial cells in coronary plaques, which may result in higher levels of adhesion molecules, proinflammatory cytokines and monocyte chemotactic proteins in the circulation during plaque rupture. Circulating plasma OX40L may pass through the damaged atherosclerotic endothelium and come into direct contact with the cells inside the lesion, increasing the inflammation of the coronary plaque.

TNC is involved in the physiological as well as the pathological remodelling of blood vessels. TNC is expressed in the heart in the early embryo, where it contributes to the development of the myocardium, valves and coronary vessels (6). In cardiovascular pathology, TNC is involved in post-MI remodelling, neointimal hyperplasia and restenosis following percutaneous transluminal coronary angioplasty and bypass grafting, as well as pulmonary vascular disease and hyperten- sion (17). Under normal conditions, only low levels of TNC are found in adult tissue. However, higher levels of TNC expression were previously reported in areas of wound healing, cancer development and cardiovascular disease, where the level of expression appears to be a reliable biomarker of disease progression and poor patient prognosis (18). Emerging evidence (6) has highlighted a number of novel roles for TNC in cardiovascular disease.

Although a previous study indicated that the OX40/OX40L pathway is crucial in atherosclerosis (19), the precise function of the OX40L system in ACS has not been fully elucidated. The present study demonstrated that the OX40L expression levels were increased in patients with ACS. The regression analysis indicated that the serum levels of TNC, which degrades the connective tissue matrix protein, ultimately resulting in plaque rupture and the development of ACS, were significantly associated with the levels of OX40L. These findings suggested that TNC and OX40L expression in patients with ACS may 
affect the development of atherosclerosis and the instability of atherosclerotic plaques. However, further studies are required to verify these findings.

Compared to patients with SA, those with UA and AMI exhibited a higher number of complex coronary lesions (20). In our study population, complex coronary stenosis morphology was found in $\sim 68 \%$ of the patients with UA, $60 \%$ of the patients with MI and $24 \%$ of the patients with chronic SA. A positive correlation between TNC and OX40L expression and the number of complex lesions was observed in patients with ACS. However, in SA patients, such a correlation was not observed. Therefore, our findings suggest that the increased expression of serum TNC and plasma OX40L in patients with ACS may be a marker of immune activation and may also be involved in the underlying pathogenic processes. The interruption of the OX40-OX40L interaction was shown to ameliorate several autoimmune-like diseases $(21,22)$, indicating that the downregulation of the OX40-OX40L interaction and TNC and OX40L expression may represent a novel therapeutic approach for patients with ACS. However, the results of this study should be interpreted with caution, due to the relatively limited patient sample. In addition, coronary angiography has certain limitations compared to angioscopy and intravascular ultrasound and further investigation is required to clarify its role in ACS.

In conclusion, our results suggest that the concentrations of serum TNC and plasma OX40L may be of value as clinical markers of the severity of CAD and the downregulation of TNC and OX40L expression may represent a novel therapeutic approach for patients with ACS. However, the precise mechanisms underlying the involvement of TNC and OX40L in ACS have not been fully elucidated. Further investigation is required to determine whether TNC and OX40L may be used as biomarkers in the clinical setting to guide intervention strategies in patients with CAD, or serve as markers under emergency conditions to diagnose ACS more efficiently.

\section{Acknowledgements}

This study was supported by the Medical Science Foundation of Wuxi, Jiangsu province (grant no. YGM1111).

\section{References}

1. Lin Y, Pan W, Ning S, Song X, Jin Z and Lv S: Prevalence and management of hypertension in patients with acute coronary syndrome vary with gender: Observations from the Chinese registry of acute coronary events (CRACE). Mol Med Rep 8: 173-177, 2013.

2. Goodacre S, Thokala P, Carroll C, et al: Systematic review, meta-analysis and economic modelling of diagnostic strategies for suspected acute coronary syndrome. Health Technol Assess 17: 1-188, 2013.

3. Hou HW, Li XG, Yan M, Hu ZQ and Song YE: Increased leukocyte Rho-kinase activity in a population with acute coronary syndrome. Mol Med Rep 8: 250-254, 2013.
4. Dobaczewski M, Gonzalez-Quesada C and Frangogiannis NG: The extracellular matrix as a modulator of the inflammatory and reparative response following myocardial infarction. J Mol Cell Cardiol 48: 504-511, 2010.

5. Ohno Y, Izumi M, Yoshioka K, Ohori M, Yonou $\mathrm{H}$ and Tachibana M: Prognostic significance of tenascin-C expression in clear cell renal cell carcinoma. Oncol Rep 20: 511-516, 2008.

6. Golledge J, Clancy P, Maguire J, Lincz L and Koblar S: The role of tenascin $\mathrm{C}$ in cardiovascular disease. Cardiovasc Res 92: 19-28, 2011.

7. Trescher K, Thometich B, Demyanets S, et al: Type A dissection and chronic dilatation: tenascin-C as a key factor in destabilization of the aortic wall. Interact Cardiovasc Thorac Surg 17: 365-370, 2013

8. Ueki T, Murata S, Kitamura N, Mekata E and Tani T: Pre-treatment with cyclophosphamide or OX40 (CD134) costimulation targeting regulatory $\mathrm{T}$ cell function enhances the anti-tumor immune effect of adoptively transferred $\mathrm{CD}^{+} \mathrm{T}$ cells from wild-type mice. Mol Med Rep 2: 615-620, 2009.

9. Chen Y, Zhang L, Huang H, et al: Association of OX40 and OX40L gene polymorphisms with acute coronary syndrome in a Han Chinese population. DNA Cell Biol 30: 597-602, 2011.

10. Willett BJ, McMonagle EL, Logan N, Schneider P and Hosie MJ: Enforced covalent trimerisation of soluble feline CD134 (OX40)-ligand generates a functional antagonist of feline immunodeficiency virus. Mol Immunol 46: 1020-1030, 2009.

11. National Committee for Clinical Laboratory Standards (NCCLS): Procedure for the Determination of Fibrinogen in Plasma; Approved Guideline. NCCLS document H30-A. 2nd edition. Wayne, PA, p99, 1999.

12. Gensini GG: A more meaningful scoring system for determining the severity of coronary heart disease. Am J Cardiol 51: 606, 1983.

13. Profumo E, Buttari B, Saso L, Capoano R, Salvati B and Riganò R: T lymphocyte autoreactivity in inflammatory mechanisms regulating atherosclerosis. ScientificWorldJournal 2012: 157534,2012

14. Nicholls SJ, Andrews J, Puri R, Uno K and Kataoka Y: Imaging progression of coronary atherosclerosis. Circ J 77: 3-10, 2013.

15. Reriani MK, Flammer AJ, Jama A, Lerman LO and Lerman A: Novel functional risk factors for the prediction of cardiovascular events in vulnerable patients following acute coronary syndrome. Circ J 76: 778-783, 2012.

16. Christenson E and Christenson RH: The role of cardiac biomarkers in the diagnosis and management of patients presenting with suspected acute coronary syndrome. Ann Lab Med 33: 309-318, 2013.

17. Niebroj-Dobosz I: Tenascin-C in human cardiac pathology. Clin Chim Acta 413: 1516-1518, 2012.

18. Ohtsuka M, Yamamoto H, Oshiro R, et al: Concurrent expression of $\mathrm{C} 4.4 \mathrm{~A}$ and Tenascin- $\mathrm{C}$ in tumor cells relates to poor prognosis of esophageal squamous cell carcinoma. Int J Oncol 43: 439-446, 2013.

19. Nakano M, Fukumoto Y, Satoh K, et al: OX40 ligand plays an important role in the development of atherosclerosis through vasa vasorum neovascularization. Cardiovasc Res 88: 539-546, 2010.

20. Koch KC, Schaefer WM, Ersahin K, et al: Haemodynamic significance of stent lesions compared to native coronary lesions: a myocardial perfusion imaging study. Heart 90: 691-692, 2004.

21. Kaur D and Brightling C: OX40/OX40 ligand interactions in T-cell regulation and asthma. Chest 141: 494-499, 2012.

22. Croft M, So T, Duan W and Soroosh P: The significance of OX40 and OX40L to T-cell biology and immune disease. Immunol Rev 229: 173-191, 2009. 